\title{
Formation of Charged Soliton in a Metal/polymer Structure Fan-Hua MENG ${ }^{\mathrm{a}}$ and Wen LIU ${ }^{\mathrm{b},{ }^{*}}$
}

\author{
Department of Physics and Information Engineering, Jining University, Qufu 273155, China \\ aemail:yygw2001@163.com, bemail:liuwenjnxy@163.com \\ *Corresponding author
}

\begin{abstract}
Keywords: Soliton, Polymer, Nonadiabatic dynamic
\end{abstract}
\begin{abstract}
Based on a nonadiabatic dynamic method, charge injection is studied in a metal\polymer structure. For a system with a short polymer chain, with a proper voltage bias applied to the metal electrode, one electron is injected into the polymer chain and then a charged soliton forms. For a system with a relatively long chain, it is hard to inject an electron to polymer to create a charged soliton. Electrons can be injected into the polymer by further increasing the voltage bias. The injected electrons form a polaron-like wave packet rather than a charged soliton. The reason lies in the requirement for charge injection that there must be some overlap between wave functions of soliton state and interfacial electrons.
\end{abstract}

\section{Introduction}

For about three decades conjugated organic materials have been attracting considerable interest $[1,2]$. The simplest among these materials is polyacetylene(PA), and the twofold degeneracy of the ground state of trans- polyacetylene leads to the formation of soliton excitations [3,4]. A lot of works have been done on soliton dynamics [5-8]. Yoshiyuki Ono et al. have studied the dynamical progress of the formation of a soliton pair developing from a photogenerated electron-hole pair in polyacetylene by adopting the SSH Hamiltonian, and concluded that the system size might affected the channel of the relaxation process [9]. They also investigated the effects of a site-type impurity and a bond-type disorder on the dynamics of charged and neutral solitons [10]. Sven Stafström did numerical simulation on the interchain charge transport of soliton [11]. They performed simulations for a two-chain system with the first chain being odd numbered initially holding a negative soliton on it. They found that only in the system where the second chain is odd numbered can realize the interchain charge transport.

Up to know, there has been no work on the charge injection from a metal electrode to a PA chain initially with a neutral soliton-type distortion. In this paper, we present our results of charge injection study in such a system. The on-site energies of both layers are assumed to be zero, which means that the Fermi level of the metal is near the middle of the band gap of the polymer, i.e. the soliton level. The model and method is briefly described in Sec. II. Our results are presented and analyzed in Sec.III. The last section is devoted to discussion and summary.

\section{Model and Methodology}

We consider a one-dimensional metal/polymer structure. The Hamiltonian is composed of three parts,

$$
H=H_{\text {ele }}+H_{\text {lat }}+H_{\text {ext }}
$$

The electronic part is described by the Su-Schrieffer-Heeger model

$$
H_{\text {ele }}=-\sum_{n}^{N} t_{n, n+1}\left(C_{n+1}^{+} C_{n}+\text { h.c. }\right)
$$

where $t_{n, n+1}$ is the hopping integral between site $n$ and $n+1$. We set $t_{n, n+1}=t_{0}$ for a metal electrode, $t_{n, n+1}=t_{0}-\alpha\left(u_{n+1}-u_{n}\right)$ for the polymer and $t_{n, n+1}=t_{m p}$ for the interfacial coupling between the metal electrode and the polymer. $u_{n}$ is the monomer displacement of site $n$ along the chain, and $\alpha$ describes the electron--phonon coupling between neighboring sites in the polymer chain. The polymer lattice is described classically by 


$$
H_{\text {lat }}=\frac{K}{2} \sum_{n}\left(u_{n+1}-u_{n}\right)^{2}+\frac{M}{2} \sum_{n} \dot{u}_{n}^{2}
$$

with $K$ being the elastic force constant of $\sigma$ bonds and $M$ the mass of a $\mathrm{CH}$ group.

The contribution from the external field (applied voltage bias and external electric field) is

$$
H_{e x t}=\sum_{n=1}^{N} V_{n}(t)\left(C_{n}^{+} C_{n}-1\right)
$$

A bias is applied to the left metal electrode for charge injection, $V_{n}(t)=V(t)$. A uniform electric field $E(t)$ is applied to the polymer, $V_{n}(t)=-|e| E(t)\left(n a+u_{n}\right)$ with $a$ being the lattice constant. During calculation the bias and the external field is smoothly turned on with the same mode.

In the presence of the electric field, the lattice configuration of the polymer should be governed by the Newtonian equations of motion

$$
M \ddot{u}_{n}=-K\left[2 u_{n}(t)-u_{n+1}(t)-u_{n-1}(t)\right]+2 \alpha\left[\rho_{n, n+1}(t)-\rho_{n-1, n}(t)\right]-e E(t)\left[\rho_{n, n}(t)-1\right]
$$

and $u_{n}$ is set to be zero for the metal electrode. Here $\rho$ is density matrix. In the mean-field approximation the elements of the density matrix are described as

$$
\rho_{n, n^{\prime}}(t)=\sum_{v} \psi_{v}(n, t) f_{v} \psi_{v}^{*}\left(n^{\prime}, t\right)
$$

here $\psi_{v}(n, t)=\left\langle n \mid \psi_{v}(t)\right\rangle$ denotes the projection of electronic state $\left|\psi_{v}(t)\right\rangle$ onto Wannier state $\langle n| \cdot f_{v}$ is the time-independent distribution function. The evolution of electronic state $\left|\psi_{v}(t)\right\rangle$ is determined by the time-dependent Schrö dinger equation

$$
i \hbar \frac{\partial}{\partial t} \psi_{v}(n, t)=-t_{n} \psi_{v}(n+1, t)-t_{n-1} \psi_{v}(n-1, t)+V_{n}(t) \psi_{v}(n, t)
$$

Equations (5) and (7) are solved using the Runge-Kutta method of order 8 with step-size control. The parameters in the equations above are those generally chosen for tPA: $\alpha=4.1 \mathrm{eV} / \AA$, $K=21.0 \mathrm{eV} / \AA^{2}, \quad M=1349.14 \mathrm{eV} \mathrm{fs}{ }^{2} / \AA^{2}, \quad a=1.22 \AA$.

\section{Results}

In this section, we perform calculations for the systems of different size with the metal electrode of 80 sites unchanged. First we consider a short PA chain containing 41 sites. The single odd numbered polymer chain bears a neutral soliton defect located in the middle of the gap, forming the so called "mid-gap" state, corresponding to the energy difference between the top of the valence band of the polymer and the soliton level of about $0.7 \mathrm{eV}$. The metal electrode has a quasi-continuum band structure and several energy levels spread over this large energy interval. The Fermi level is about $0.1 \mathrm{eV}$ lower than the soliton level in the absence of the external field, as is shown in Fig.1(a). After the bias is turned on the energy levels of the metal electrode are lifted, which contributes to the charge injection into the polymer. 

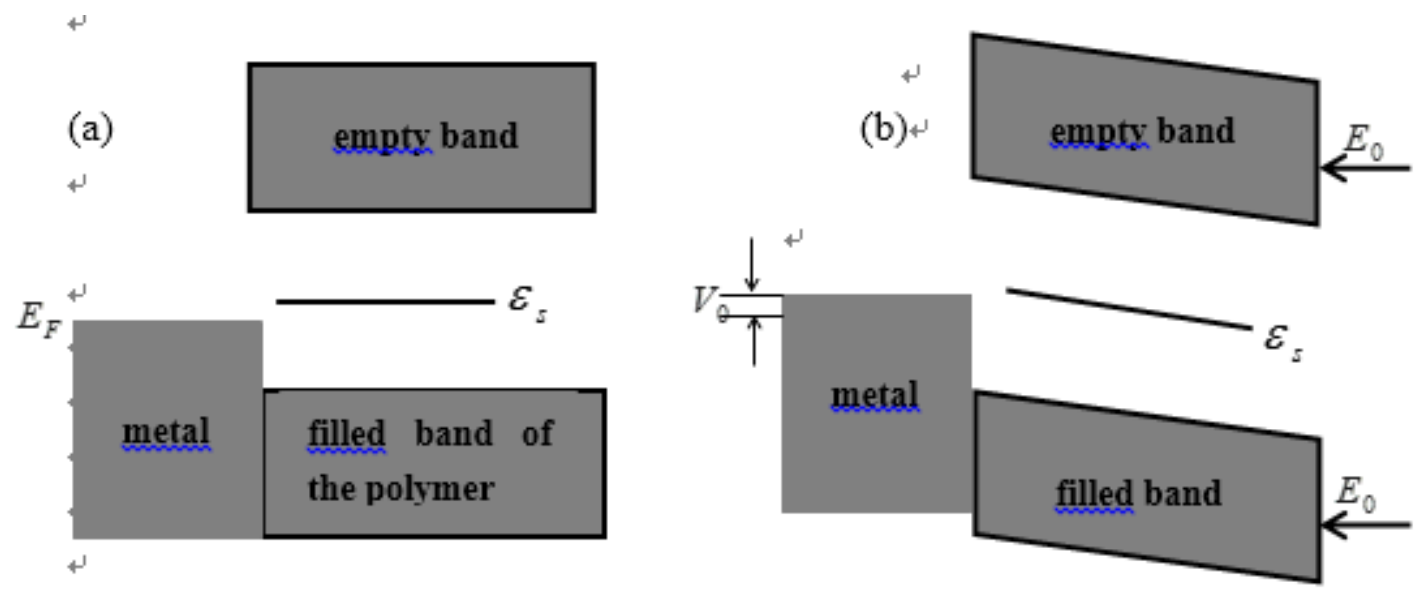

Fig.1. Sketch of the band structure of the metal electrode and polymer chain, in the absence (a) and presence (b) of the external field.

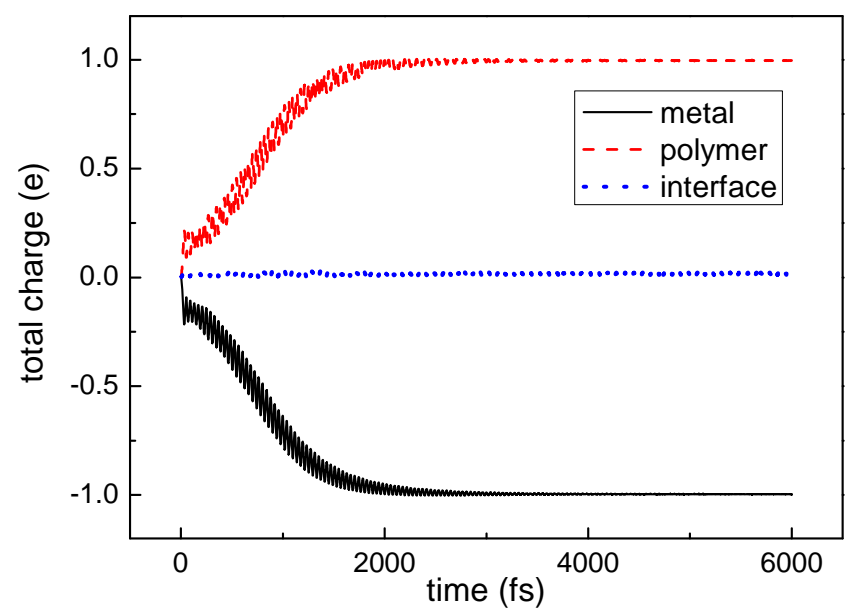

Fig.2. Evolution of the total charges in the polymer chain (dash line), metal electrode (solid line), and the charge confined to the interface (dot line) at the electric field $\mathrm{E} 0=10-4 \mathrm{~V} / \mathrm{cm}$ and $\mathrm{V} 0=0.2 \mathrm{~V}$.

The evolution of the total injected charges is presented in Fig.2. It takes about 2000fs to reach a stable state at the bias of $\mathrm{V} 0=0.2 \mathrm{~V}$ and electric field $\mathrm{E} 0=10-4 \mathrm{~V} / \mathrm{cm}$. For the present bias, one electron can be injected and about 0.02e are bound to the interface. We can know better the injection process by the injected charge distribution $\rho_{n, n}$ and staggered order parameter $y_{n}=(-1)^{n+1} / 4\left(u_{n+1}+u_{n-1}-2 u_{n}\right)$. Fig.3 shows $\rho_{n, n}$ and $y_{n}$ at several typical times. As the bias is added the Fermi level goes up towards the soliton level and charges begin to be injected into the polymer through the interface. Initially, at $\mathrm{t}=0 \mathrm{fs}$ the polymer chain is neutrally charged and there is no net charge distribution. At $\mathrm{t}=658 \mathrm{fs}$ the quantity of the injected charges accumulates to $0.5 e$, which forms a small wave packet, see Fig.3(a). The lattice configuration doesn't undergo obvious change compared with original configuration of the neutral soliton. We know that a charged soliton has the same lattice configuration as a neutral one does. So it is reasonable to think that the charges injected into the polymer forms a "small charged soliton" with charge smaller than $1 e$. As time goes on the injected charges increase to1e, i.e. one electron escapes the Fermi level to occupy the soliton level, therefore a negatively charged soliton forms in the polymer, as can be seen in Fig.3(b) which shows the charge distribution and lattice configuration of the polymer at $t=2200 \mathrm{fs}$. It should be noted that the polymer chain here we adopt is a short one. This small size restricts the motion of the charged soliton. 


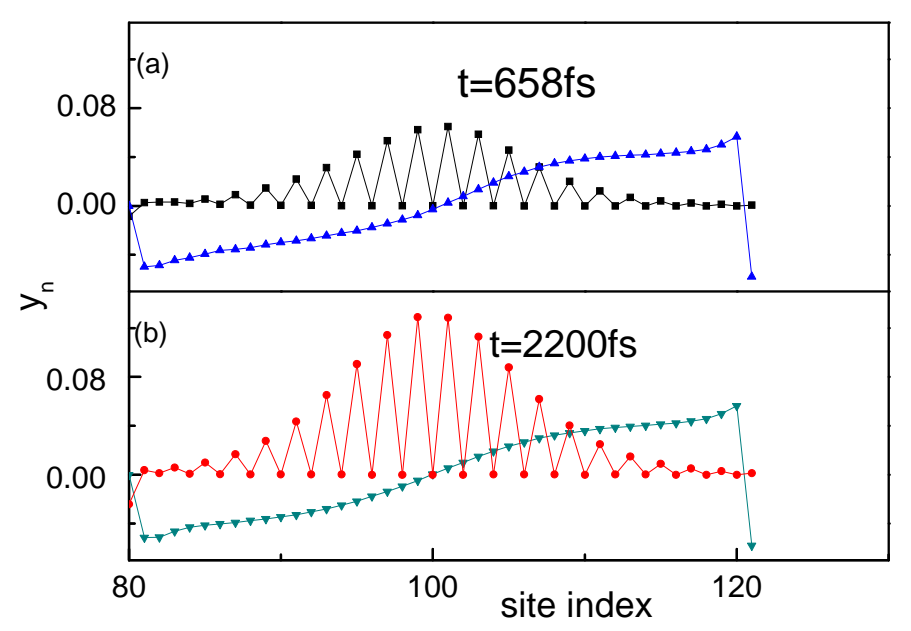

Fig.3. Injected charge distribution and staggered order parameter at two typical times (a) $t=658 \mathrm{fs}$ and (b) $t=2200 \mathrm{fs}$.

Now we turn to a relative long polymer chain containing 101 sites. We have calculated the energy levels of the system in the absence of the external field and find that the energy difference between Fermi level and soliton level is also $0.1 \mathrm{eV}$. We provide the same external field as above. However, the calculations show that no electron can be injected into the polymer at all. It can be understood from Fig.4, which shows the wave function of the soliton level. Although we have fixed the center of the original soliton defect near the interface to make injection easy, after interation the center goes to the 111st site, i.e. there is no overlap between wave functions of the soliton level and electrons at the interface. Electrons can't go to the polymer from the metal electrode. This is different form an isolated polymer chain in which the center of the soliton can be fixed as required. The main reason may consist in the asymmetry of such a metallpolymer structure. As a matter of fact, we further increase the bias to $0.73 \mathrm{eV}$, when the Fermi level approaches the lowest unoccupied state of the polymer, charges starts to be injected into polymer. At about $t=250 \mathrm{fs}$ the system reaches a dynamical balance and there are1.3e injected into the polymer. Fig.5 shows the lattice configuration at $t=1989 f s$. These injected charges accumulate to form a polaron-like wave packet.

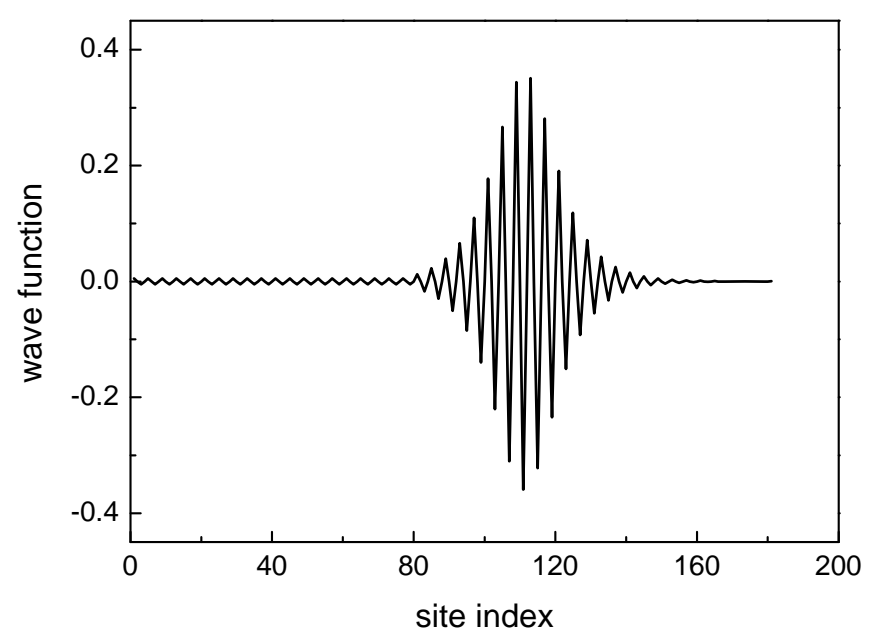

Fig. 4. Wave function of the soliton level. 


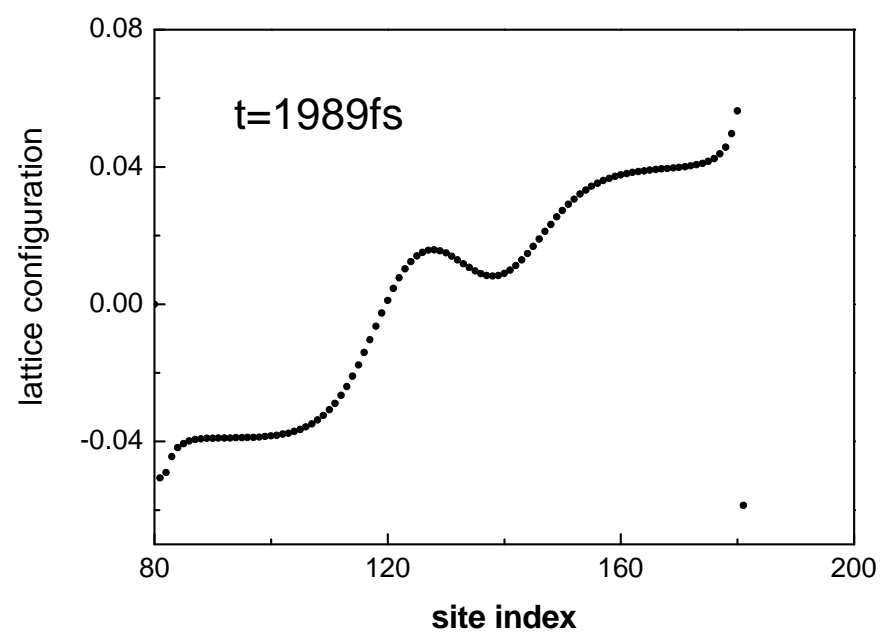

Fig.5. The lattice configuration of the polymer chain at $V_{0}=0.73 \mathrm{~V}$ and $E_{0}=5 \times 10^{-5} \mathrm{~V} / \mathrm{cm}$.

\section{Summary}

In this work, we have investigated the dynamical process of charge injection from a metal electrode to a polymer chain with a soliton-type distortion. We performed simulations for systems of different size with no excessive charge. Charge injection into soliton level in such a system requires not only a sufficient bias but also overlap between wave functions of soliton level and interfacial electrons. The results show that one electron can be injected into a short polymer chain to create a charged soliton at a small bias. But it is hard to realize in a long polymer chain because there is no overlap in the initial state obtained by minimizing the total energy of the tPA chain. Electrons can be injected into polymer when the Fermi level approaches the lowest unoccupied state by further increasing the bias

\section{References}

[1] J. H. Burroughes et al., Nature (London) 347, 539 (1990).

[2] S. Datta and W. Tian, Phys. Rev. B 55, R1914 (1997).

[3] W. P. Su, J. R. Schrieffer, and A. J. Heeger, Phys. Rev. Lett. 42, 1698 (1979).

[4] M.J.Rice, Phys. Lett. 71A, 152 (1979).

[5] Y. Ono and A. Terai, J. Phys. Soc. Jpn. 59, 2893 (1990).

[6] Y. Ono, M.Kuwabara and A. Terai, J. Phys. Soc. Jpn. 60, 3120 (1991).

[7] A.R.Bishop, D.K.Campbell, P.S.Lomdahl, B.Horovitz, and S.R.Phillpot, Synth. Met. 9, 223 (1984).

[8] M.Kuwabara, Y. Ono and A. Terai, J. Phys. Soc. Jpn. 61, 2412 (1992).

[9] Y. Hirano and Y. Ono, J. Phys. Soc. Jpn. 67, 3835 (1998).

[10] M.Kinoshita, Y. Hirano, M.Kuwabara and Y. Ono, J. Phys. Soc. Jpn. 66, 703 (1997).

[11] A. Johansson and S. Stafstrom, Phys. Rev. B 65,045207 (2002). 